\title{
LA DESIGUALDAD POLÍTICA ANTE LA CRISIS DEL ESTADO SOCIAL
}

\author{
POLITICAL INEQUALITY IN THE CRISIS OF WELFARE STATE
}

\author{
Manuel Menéndez Alzamora \\ Universidad de Alicante. España/Spain \\ manuel.menendez@ua.es
}

Recibido/Received: 02/04/2013

Aceptado/Accepted: 20/06/2013

\section{RESUMEN}

El texto parte de la fuerte conexión entre Estado social fuerte y las políticas públicas activas de distribución y equilibrio de la riqueza. Desde esta premisa se describen algunos escenarios en los que las desigualdades estructurales impiden el desarrollo de políticas públicas activas. De manera específica se alude a determinados datos relativos a America Latina. Estos datos se presentan como contrapunto al contexto europeo. Por último, se incide en el papel de las clases medias como destinatarias de las nuevas políticas públicas necesarias para dar un nuevo sentido al estado social del siglo XXI.

\section{PALABRAS CLAVE}

Políticas públicas, igualdad, Estado social, clases medias.

\section{SUMARIO}

1. Políticas públicas y crisis del Estado social. 2. Políticas públicas y desigualdad social. 3. Clases medias y nueva cohesión política y social. Bibliografía.

\begin{abstract}
The text part of the strong connection between strong Welfare State, and the active Public Policies of equilibrium, and distribution of wealth. From this premise we consider some scenarios in which structural inequalities prevent the development of active Public Policies. Specifically it refers to certain data from Latin America. These data are presented as a counterpoint to the European context. Finally, it deals with the role of the middle classes as recipients of new policies needed to give a new meaning to the social status of the XXI century
\end{abstract}

\section{KEYWORDS}

Public policies, equality, Welfare State, middle classes.

\section{CONTENTS}

1. Public policy and crisis of the Welfare State. 2. Public policies and social inequality. 3. Middle classes and a new political and social cohesion. References. 


\section{POLÍTICAS PÚBLICAS Y CRISIS DEL ESTADO SOCIAL}

La idea de la que partimos es la que vincula las políticas públicas sociales a la presencia de un Estado fuerte y, desde este punto de vista, todo Estado fuerte necesita para su arraigo e implementación que su política económica tenga una dimensión democrática y social. Esto significa claramente que todo Estado democrático fuerte debe tener entre sus misiones principales la de promover la función redistributiva. Esta función cumple sus objetivos cuando se produce un balance equilibrado entre los ingresos recibidos por el Estado, vía impuestos, y los gastos sociales. Esta conexión de las políticas públicas con la función redistributiva del Estado nos conduce hacía la Hacienda Pública y el sistema fiscal como núcleo del que parten todas las políticas distributivas.

Las políticas fiscales son la herramienta fundamental de la que las políticas públicas se sirven para cumplir la misión distributiva. En el plano internacional la fiscalidad sobre determinadas actividades suele vincularse con ese sentido distributivo, así por ejemplo se observa en los debates sobre la tasa Tobin, el llamado impuesto sobre las transacciones financieras, y en las cuotas sobre las emisiones de gases con efecto invernadero. En el ámbito interno dicha cuestión se plantea cuando debatimos sobre la legitimidad de la aplicación de ciertas tasas o copagos, en este caso se trata de verificar si estas tasas sirven para universalizar la prestación de servicios públicos fundamentales, como lo son la justicia y la sanidad o, por el contrario, son meras tasas con una finalidad exclusivamente recaudatoria.

Con el objetivo de que en un Estado se produzca un equilibrio positivo entre la recaudación de impuestos y su aplicación distributiva en forma de gastos sociales son necesarios dos elementos fundamentales. Elementos muy incardinados con la construcción de un Estado social fuerte. En primer lugar es necesario que la esfera pública se levante sobre un orden institucional sólido y consolidado. Sin instituciones políticas que vertebren la acción política en la esfera pública es imposible construir un auténtico Estado social. Las instituciones públicas y sus burocracias son el cauce organizativo necesario sin el cual la redistribución deviene, de facto, en imposible.

En segundo lugar, todo Estado fuerte necesita de una cultura política con un fuerte arraigo de ciertos valores como el de cohesión y el de solidaridad social. En el plano de los intangibles sociales las sociedades necesitan de valores fuerza que legitiman socialmente el trasfondo y las finalidades de las políticas públicas.

Hasta este punto hemos planteado en líneas generales las bases sobre las que debe asentarse un Estado social fuerte. Ahora bien, la realidad que nos acompaña muestra serias diferencias ante lo que la teoría nos propone. Podemos indagar en estas diferencias en un doble plano, primero teniendo como referencia el difícil camino de las naciones de América Latina en punto a transformar sus estructuras estatales; en segundo lugar, el plano referido a Europa, cuna tradicional del Estado social, pero donde, a partir de 2008, la profunda crisis ha transformado y reorientado el modelo social de Estado.

A partir de este doble referente, el latinoamericano y el europeo, vamos a analizar aquellos elementos que sirven para interrogarnos sobre la vigencia y futuro del Estado social en un momento de crisis y transformación negativa. Los tres elementos clave que sirven para descifrar el momento actual del Estado social son, en primer lugar, el problema de la desigualdad; en segundo lugar, la cuestión de la cohesión social y, en tercer lugar, el problema de la deslegitimación institucional. 


\section{POLÍTICAS PÚBLICAS Y DESIGUALDAD SOCIAL}

El problema de la desigualdad incide vertebralmente en la configuración de todo Estado social. En Estados con desigualdades estructurales, con barreras sociales infranqueables, las políticas públicas de redistribución tienen una alta probabilidad de fracaso. Cualquier función de redistribución social necesita para su implementación de cierta porosidad social. Cuando los desequilibrios económicos y sociales polarizan la sociedad creando estratos sociales aislados, la función de recaudación y distribución del Estado es impracticable. En estas situaciones sólo cabe la ayuda social, vía interna o vía externa. América Latina es una de las regiones más desiguales del planeta y por ello escenario de eso que en ocasiones se denomina "sociedad archipiélago", metaforizando un escenario en el que sobre masas oceánicas de extrema pobreza sobresalen minúsculos territorios de extrema riqueza, esto es, una sociedad construida sobre extremos sociales. Dos siglos de modernización a todos los niveles post-independencia no han permitido avanzar en la configuración de Estados en donde impere cierta igualdad social. Como señala Cordera Campos: "La relación del quintil más rico $(20 \%)$ con el más pobre en América Latina es de 17 veces, en tanto que en los países G-7 llega a siete. Si, además de ello, se observan las distancias entre el decil (10 por \%) más rico y el más pobre, la brecha es mayor: 34 veces contra 12" (Cordera, 2012:40).

La desigualdad social tiene, como indicaba, dos frentes en la sociedad, uno es la imposibilidad de circulación económica entre clases, esto es, el aislamiento de las clases sociales. El segundo elemento es el de la polarización: en el caso de México, Cordera Campos, utilizando los Indicadores del Desarrollo Mundial de 2010 del Banco Mundial, indica que: "En 2008 al $10 \%$ más pobre de los mexicanos les correspondió 1,2\% del ingreso nacional total, y que el $10 \%$ más rico de la población concentró $41,3 \%$ de la renta nacional total" (Cordera, 2012:42).

La desigualdad social tiene en América Latina un factor añadido a considerar: la concentración de las bolsas sociales de pobreza en los colectivos y grupos indígenas. En el caso de México, Vázquez Gutiérrez, utilizando los indicadores de medición de pobreza del Consejo Nacional de Evaluación de la Política de Desarrollo Social (CONEVAL), señala que "un 44,2 \% de la población total se sitúa dentro de algún nivel de pobreza, en tanto un $11,2 \%$ se encuentra en situación de pobreza multidimensional extrema. De entre estos últimos, el $77 \%$ son indígenas". (Vázquez Gutiérrez, 2012:49-50). Como apunta Milanovic: "El Estado del bienestar se edificó sobre la presuposición de la homogeneidad étnica y cultural de la población. La homogeneidad no sólo incrementa la afinidad entre diferentes segmentos de la población, sino que garantiza que todos más o menos siguen normas sociales similares. Si nadie engaña haciéndose pasar por mayor con el fin de obtener una pensión o cogiendo la baja por enfermedad cuando no está enfermo, el Estado de bienestar es autosostenible. Pero si estas normas no son observadas por todos, se desmorona" (Milanovic, 2013:47-48).

Este escenario no es exclusivo de regiones con desequilibrios endémicos, Navarro observa la presencia de esta desigualdad estructural en España, y afirma: "las desigualdades sociales también disminuyeron durante los años ochenta y principios de los años noventa, aunque, de nuevo, no lo suficiente para evitar que España continuara siendo de los países con más desigualdades de renta de la UE” (Navarro, 2002:105)

En estas condiciones los flujos de redistribución están bloqueados por murallas insalvables, estamos ante un bloqueo social que no permite la interacción entre clases 
sociales. La desigualdad y su manifestación en el formato de "sociedad archipiélago" tiene dos consecuencias letales, una es el aumento de los índices de pobreza y otra es la deslegitimación del orden social dentro del cual se ha gestado esta polaridad. Esta deslegitimación, que abordaremos en el tercer punto relativo a las instituciones políticas, se manifiesta palmariamente en el descrédito democrático y en la sensibilización favorable hacia los populismos. Bastidas Colinas, utilizando los datos del Latinobarómetro 2011, señala que: "el $73 \%$ de los mexicanos dice sentirse insatisfecho con la democracia y sólo el $23 \%$ dice sentirse satisfecho. El $40 \%$ dice en México que la democracia es la mejor forma de gobierno, el $14 \%$ que un gobierno autoritario puede ser preferible, pero al $36 \%$ de la población, que le da lo mismo tener un régimen democrático o no. Preocupante" (Bastidas Colinas, 2012:27).

En tercer lugar, y en conexión con lo anterior, debemos cerrar la secuencia afirmando que la polarización social y la desigualdad acaban afectando a la salud de la vida democrática y golpeando en la legitimación de la vida institucional. Cuando las instituciones políticas del Estado son ineficaces en punto a establecer ciertos niveles de igualdad social, los ciudadanos se apartan políticamente de estas instituciones, rompiéndose todo principio de cohesión social. Si esta cohesión social debe expresarse a través del aparato institucional del Estado en clave de adhesión al mismo, la desmembración de una conduce al bloqueo del otro.

En estas situaciones de crisis institucional el Estado se desdobla en Subestados a la sombra, en Estados paralelos, en un proceso de desvertebración que arranca en el ámbito económico y contamina como una mancha el resto de capas de la esfera pública. Las manifestaciones más habituales de la aparición de estos subsistemas políticos son la corrupción y, de manera más agravada en América Latina, las redes del crimen organizado.

Los fenómenos de corrupción económica tienen en este sentido mucha relación con los procesos de empobrecimiento y desaparición de las clases medias. El surgimiento de mercados paralelos en los que se comercia fuera de los circuitos legales y sin obligaciones fiscales, son una manifestación profunda del descrédito institucional provocado por desigualdades económicas sociales radicales.

\section{CLASES MEDIAS Y NUEVA COHESIÓN POLÍTICA Y SOCIAL}

El problema de la distribución de la riqueza está unido al nacimiento y desarrollo de las clases medias. La segunda mitad del siglo XIX es el momento en el que converge el naciente impulso de la sociedad de masas con las primeras políticas de solidaridad social a través de los impuestos.

Las clases medias observarán esa primera etapa de desarrollo desde la década de los 80 del siglo XIX hasta la primera Guerra Mundial y, en una segunda fase, después de la segunda Guerra Mundial, durante tres décadas que alcanzan hasta la mitad de la década de los 70 en un momento de esplendor de la expresión social y económica de la clase media. En este primer momento de aparición de las clases medias burguesas la idea de solidaridad distributiva resulta fundamental. Como afirma Rosanvallon, la expresión de Léon Bourgeois, autor de Solidarité, "el hombre solo no existe", es la idea síntoma que impulsó en toda Europa las corrientes intelectuales que fueron base para la teorización de las políticas sociales que impulsaron socialistas de cátedra en Alemania, fabianos y nuevos 
liberales en Gran Bretaña y republicanos solidaristas en Francia (Rosanvallon, 2010:260261).

La idea de solidaridad y distribución nacen ligadas a la aparición de los impuestos progresivos. Como el propio Léon Bourgeois señalaba para explicar el fundamento de los impuestos, toda persona que nace tiene una deuda con la sociedad que le ha precedido a lo largo de la historia, por tanto, los impuestos progresivos sirven para devolver y pagar esta deuda social. En consecuencia, el fundamento redistributivo tendría un sentido histórico, de ajuste de cuentas con el pasado. En la dirección de lo que Alfred Fouillée describía en su obra La Propriété sociale et la démocratie, como la "justicia reparativa" (Rosanvallon, 2010:262-263).

De esta manera se configura la formulación teórica de las reformas fiscales antes de la I Guerra Mundial. En Estados Unidos los grandes teóricos de la reforma fiscal, Richard T. Ely y Edwin R. A. Seligman, son partidarios del impuesto progresivo redistributivo a modo de contrapeso de la individualización de los mecanismos de mercado. Como señala de manera sintética Rosanvallon, en este momento se produce una superposición entre la noción de solidaridad en el orden socio-económico y la noción de ciudadanía en el orden político, "la sociedad estaba doblemente compuesta como un cuerpo" (Rosanvallon, 2010: 264). Bajo este paradigma de la sociedad entendida como cuerpo social es muy fácil entender la aparición de conceptos como el de "garantía de un mínimo de existencia", teorizado en Francia por Fouillée y Bourgeois (Rosanvallon, 2010:264-265).

Las bases de esta política redistributiva sufrieron un profundo impacto derivado de la I Guerra Mundial. Hasta este momento esta idea de igualdad distributiva convivió con un ideal democrático en el que la autonomía del individuo burgués y su diferenciación formaban parte de ese mismo sustrato cultural que fundamentaba la redistribución. En el periodo de entreguerras asistiremos al intento de eliminar la diferenciación y sustituirla por la homogeneidad. Señala Rosanvallon que los intentos de democracia homogénea son la base de la propuesta de Carl Schmit. En este caso la homogeneidad natural proporcionará una especie de igualdad de la misma naturaleza y, por tanto, más allá de cualquier política de redistribución de lo que realmente se trata es de practicar políticas de homogenización, el Estado social deja de tener sentido como redistribuidor fiscal para convertirse en un mero bloque-masa unido e indistinto (Rosanvallon 2010:272-273).

Desde mitad de los años 1970 y a partir de los años 1980, al final de los "treinta años gloriosos" el Estado social distribuidor entra en una crisis de deslegitimación, que como plantea Rosanvallon es un proceso de deslegitimación que nos retrotrae a los momentos del siglo XIX previos al desarrollo de las políticas de redistribución fiscal.

Pierre Hassner comenta la propuesta de Moisés Naím sobre el conflicto entre las dos clases medias que existen actualmente. Por un lado las clases medias de Occidente, sumidas en una profunda decadencia cuyo origen están en la falta de productividad, "que obedecen a su vez a los avances de la tecnología, a la competencia de los bajos salarios y a leyes sociales laxas o inexistentes en los países emergentes y a la codicia y a la búsqueda de ganancias a corto plazo, gracias a la especulación y las deslocalizaciones" (Hassner, 2013: 28). Por otro lado, las clases medias de los países emergentes también viven bajo la crisis y en estado de frustración entre otras razones porque "no encuentran salidas acordes con su formación o bien tienen aspiraciones políticas y culturales que topan con las estructuras tradicionales, oligárquicas de su país, de modo que miran hacia la emigración o la revolución" (Hassner, 2013:28). El resultado final no será un choque entre estados de carácter militar o violento la probabilidad es que la lucha entre las clases medias se 
produzca, "pero entre grupos, entre nómadas y sedentarios, entre emigrantes en busca de refugio o de un atisbo de esperanza y residentes que vean en ellos peligrosos rivales o enemigos" (Hassner, 2013:31).

La función de estas clases medias resulta central y para el caso español, Navarro afirma: "En España no ha cristalizado todavía la alianza de la clase trabajadora con las clases medias, alianza básica para el desarrollo de un Estado de bienestar de calidad (...). El reto en España, por lo tanto, es diseñar un Estado de bienestar en el que las clases medias, que tiene unas expectativas más elevadas, se encuentren cómodas" (Navarro, 2002:157).

La crisis de las clases medias se nos presenta con fuertes daños en la base de su identidad. Bouzou no considera que las clases medias de Occidente vayan a desaparecer, pero sí constata que su número y su representación política disminuyen, "lo que se traduce en una crisis de identidad que puede tener dañinas consecuencias políticas" (Bouzou, 2013:25).

Punto clave en esta ardua tarea de recomposición de la identidad de esas clases medias es una renovada concepción de los servicios públicos como fundamento del Estado social. Señala Ginsbourger como el modelo anglosajón de servicio público que ha tenido fuerte impronta durante las dos últimas décadas, el llamado "modelo mercantil", caracterizado por identificar al ciudadano con el cliente debe superarse en la actualidad por un nuevo concepto de servicio público, un denominado "modelo cívico", en el que tengan fuerza las nociones de interés general y gratuidad. Este cambió de dirección debe acometerse, además, en un escenario que para Ginsbourger, siguiendo los modelos de registro profesional del sociólogo Yves Lichtenberger, está dominado por un ejercicio de las actividades laborales caracterizado por redes de actuación imprevisible o cambiante. En otras palabras, la prestación de servicios públicos en el siglo XXI ha devenido una actividad muy dinámica por la pluralidad de destinatarios y por la movilidad de los mismos. Y ello afecta profundamente y obliga a redefinir las funciones de los prestatarios de los servicios públicos. Apunta directamente hacia una nueva impronta que afecta a la naturaleza del Estado social.

\section{BIBLIOGRAFÍA}

BASTIDAS COLINAS, S. (2012), "La buena democracia con algo de acidez", La Vanguardia dossier, $\mathrm{n}^{\circ} 44$, pp. 23-27.

BONFIL BATALLA, G. (1987), México profundo: una civilización negada, México, Grijalbo. BOURGUIGNON, F. (2012), La mondialisation de l'inégalité, París, Seuil.

BOUZOU, N. (2013), "¿Qué políticas públicas son posibles, cuando las clases medias desaparecen?", La Vanguardia dossier, n 47, pp. 21-25.

CORDERA CAMPOS, R. (2012), "La desigualdad mexicana: una marca histórica", La Vanguardia dossier, ${ }^{\circ} 44$, pp. 40-43.

GAGGI, M. y NARDUZZI, E. (2009), El fin de la clase media y el nacimiento de la sociedad de bajo coste, Barcelona, Lengua de Trapo.

GINSBOURGER, F. (2013), "Réinventer la relation de service public", Esprit, no 392, pp. 80-93.

HASSNER, P. (2013), "Del declive al conflicto", La Vanguardia dossier, n 47, pp. 26-31.

LOAEZA, S. y PRUD'HOME, J.F. (coords.) (2010), Los grandes problemas de México. Instituciones y procesos políticos (Vol. XIV), México, El Colegio de México.

MILANOVIC, B. (2012), Los que tienen y los que no tienen, Madrid, Alianza.

MILANOVIC, B. (2013), “Se ha acabado el periodo especial del capitalismo?”, La Vanguardia dossier, ${ }^{\circ} 47$, pp. 45-49. 
NAIM, M. (2011), "The Clash of the Middle Classes", The Hufffington Post, 5/8/2011.

NAVARRO, V. (2002), Bienestar insuficiente, democracia incompleta, Barcelona, Anagrama. ROSANVALLON, P. (2010), La société des égaux, París, Seuil.

UÑA JUAREZ, O. y GUTIERREZ RESA, A. (2010), Integración de los inmigrantes a través de los servicios sociales en la Comunidad de Madrid, Madrid, UNED.

VÁZQUEZ GUTIÉRREZ, J. P. (2012), "Los dilemas del multiculturalismo: retos para construcción de una perspectiva intercultural", La Vanguardia dossier, n 44, pp. 47-50.

\section{Breve currículo}

\section{Manuel Menéndez Alzamora}

Licenciado y Doctor en Derecho: Universitat de València. Autor de La generación del 14 (2006) con el Premio Nacional de la Asociación Española de Ciencia Política (AECPA) al mejor libro editado en 2006 y el XI Premio Nacional Ángel Herrera 2007 al mejor trabajo de investigación. Editor y coautor de: Sobre el poder (2007) y Participación y representación política (2008), Historia de la Teoría Política (1995), Política de la vitalidad (1996) y Política y sociedad en José Ortega Gasset: En torno a "Vieja y nueva política" (1997). Autor de artículos en revistas especializadas y trabajos en actas de congresos nacionales e internacionales. Profesor de asignaturas como: "Teoría Política", "Fundamentos de Ciencia Política", "Pensamiento Político Contemporáneo", "Libertades Públicas" y "Derecho de la información" en: Politécnica de Valencia, Universidad CEUCardenal Herrera y actualmente en la Universidad de Alicante. 